Three-dimensional free convection in molten gallium

Juel, A. and Mullin, T. and Ben Hadid, H. and Henry, D.

2001

MIMS EPrint: 2006.244

Manchester Institute for Mathematical Sciences

School of Mathematics

The University of Manchester

\footnotetext{
Reports available from: http://eprints.maths.manchester.ac.uk/

And by contacting: The MIMS Secretary

School of Mathematics

The University of Manchester

Manchester, M13 9PL, UK
} 


\title{
Three-dimensional free convection in molten gallium
}

\author{
By A. JUEL ${ }^{1}$, T. MULLIN ${ }^{1}$, H. BEN HADID ${ }^{2}$ AND D. HENRY \\ ${ }^{1}$ Schuster laboratory, The University of Manchester, Manchester M13 9PL, UK \\ ${ }^{2}$ Laboratoire de Mécanique des Fluides et d'Acoustique UMR 5509, \\ Ecole Centrale de Lyon/Université Claude Bernard Lyon 1, \\ ECL, BP 163, 69131 Ecully Cedex, France
}

(Received 22 March 1999 and in revised form 11 December 2000)

Convective flow of molten gallium is studied in a small-aspect-ratio rectangular, differentially heated enclosure. The three-dimensional nature of the steady flow is clearly demonstrated by quantitative comparison between experimental temperature measurements, which give an indication of the strength of the convective flow, and the results of numerical simulations. The three-dimensional flow structure is characterized by cross-flows which are an order of magnitude smaller than the main circulation, and spread from the endwall regions to the entire enclosure when the Grashof number is increased beyond $G r=10^{4}$. The mergence of these effects in the centre of the enclosure leads to a complex central divergent flow structure which underpins the observed transition to oscillatory convection.

\section{Introduction}

Sidewall convection in liquid metals is an important problem in the semiconductor crystal growing process known as the Bridgman technique. In this a crucible of molten material is slowly drawn from a furnace and solidification takes place. This technique is of significant practical importance in the growth of high-quality materials for optoelectronic applications as reviewed by Hill (1998). The industrial process may involve dendrite growth and the distribution of dopants and is thus a complicated problem. However, insights can be gained from studying the basic fluid dynamics that result from the differential heating of the sample since other processes are strongly influenced by the induced motion. For small temperature differences, the convection is steady and primarily consists of a large, single circulation. The bulk flow in a confined cavity evolves considerably for larger values of the driving force, as the interaction between the different regions of flow becomes significant. Thus, the mechanisms underlying the transitions to time-dependent and eventually turbulent flow are often complex. We refer to the review article by Müller \& Ostrogorsky (1993) for a discussion of convective effects in crystal growth.

Our model Bridgman configuration consists of a rectangular, insulated enclosure of square cross-section where the ends are conducting. It contains liquid gallium and is heated and cooled in a controlled way at the two opposite ends. A schematic diagram of the geometry is presented in figure 1 . We have chosen to investigate this relatively narrow configuration because of its practical relevance and we find that cross-flows are important since the onset of time dependence is essentially different to that studied in two-dimensional models. 


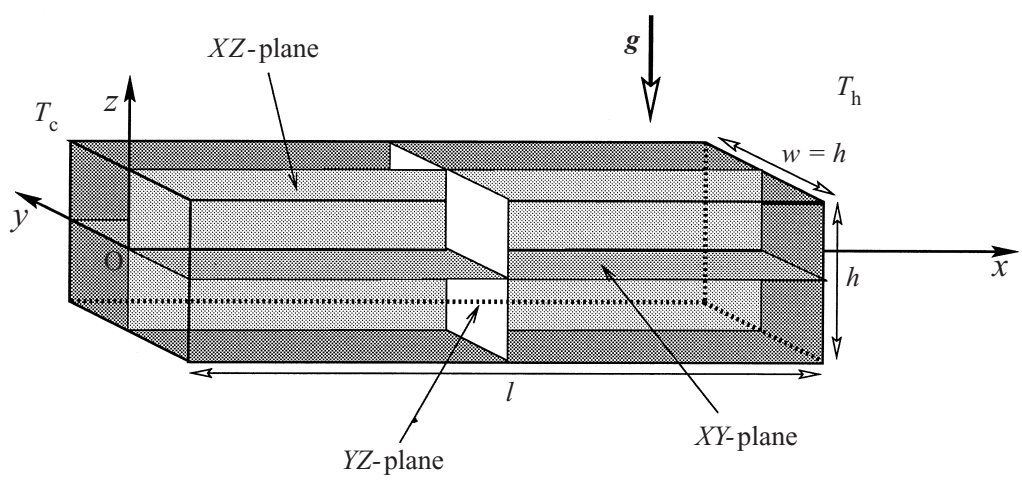

FIgURE 1. Schematic diagram of the geometry. The $X Y$-, $Y Z$ - and $X Z$-planes correspond to the central cross-sections orthogonal to the $z-, x$ - and $y$-axes respectively.

The main parameter that governs the convective flow is the Grashof number, $G r$, which measures the relative importance of buoyancy and viscous forces, and hence is proportional to the applied temperature difference. The aspect ratios, $A_{x}$ and $A_{y}$, correspond respectively to the length and width of the enclosure scaled by its height and they are chosen so that the enclosure has relative dimensions of $4 \times 1 \times 1$. The metal gallium is liquid above $29.8^{\circ} \mathrm{C}$ and its Prandtl number, $\operatorname{Pr}$, is very small with a typical value of $P r \approx 2.5 \times 10^{-2}$. Hence, most of the heat transport takes place by conduction.

Initial theoretical research on this problem is reported by Hart (1972) who derived an analytical solution for the simplified problem of parallel flow in an infinite layer of fluid, subject to a horizontal temperature gradient. This solution is commonly referred to as the Hadley circulation. Cormack, Leal \& Imberger (1974) extend Hart's approach and calculate an asymptotic solution for the limiting case of a shallow two-dimensional cavity, where $\epsilon=A_{x}^{-1}$ tends to zero. They find a buoyancy-driven, parallel flow which is moderated by viscous effects along the length of the cavity. In addition, the role of the end regions is limited to the recirculation of the fluid.

Cormack et al.'s approach is developed further by Bejan \& Tien (1978a) for the more general case of a two-dimensional cavity of finite aspect ratio. They investigate three different flow regimes which are encountered for increasing values of the Rayleigh number, $R a=G r P r$. In the conductive regime, the flow is parallel and satisfactorily described by the solution of Cormack et al. (1974). In the intermediate regime, the region over which the flow is parallel shrinks in length. The temperature profile between the endwalls is linear with a rise and fall close to the hot and cold endwalls respectively. The boundary layer regime takes place for large values of the Rayleigh number, where the greatest changes in temperature are confined to thin boundary layers adjacent to the endwalls. Bejan \& Tien $(1978 b)$ consider a slow convective flow in a horizontal, cylindrical enclosure, where differential heating is applied between the endwalls, and obtain an analytical expression for the three-dimensional flow. Interesting three-dimensional second-order effects arise from the coupling between temperature and velocity field as a result of the non-slip boundary conditions on the walls. These analyses assume a Prandtl number of order unity or larger, whereas Hart (1983a) shows that the existence of parallel flow in a two-dimensional geometry is restricted by the onset of secondary vortices at $G r \approx 4.8 \times 10^{3}$ for $A_{x}=4.0$ and $\operatorname{Pr}=2.5 \times 10^{-2}$. His work is extended by Daniels, Blythe \& Simpkins (1987) 
who determine the range of Rayleigh and Prandtl numbers where this imperfect bifurcation takes place.

Existing theoretical predictions of the stability of the flow are derived from twodimensional models. The linear stability of the Hadley circulation to both transverse and longitudinal disturbances is considered by Hart $(1972,1983 b)$ and to longitudinal disturbances by Gill (1974). The results of Hart are refined and extended by Laure (1987), Laure \& Roux (see Roux 1990) and Kuo \& Korpela (1988) who find that the first transition is to a steady, transverse roll solution for $\operatorname{Pr}<3.4 \times 10^{-2}$. Laure (1987) also performs a weakly nonlinear stability analysis and shows that the co-rotating rolls solution is locally stable.

The simulation of convective flow in a cavity of aspect ratio $A_{x}=4.0$ and for Prandtl numbers $P r=0$ and $1.5 \times 10^{-2}$ was proposed as a 'benchmark' exercise for the GAMM workshop reported by Roux (1990). For both values of the Prandtl number, the first transition is to stationary, co-rotating cells. Multiple flows are encountered as the Grashof number is raised which include one, two and three co-rotating-cell states. In addition, the flow is found to lose stability via a Hopf bifurcation, where the solution interchanges between a one-cell and three-cell state, as discussed by Winters (see Roux 1990).

Some contributions to the GAMM workshop include three-dimensional calculations for $A_{x}=4.0$ and $A_{y}=1.0,2.0$. These are by Extremet et al., Chabbard \& Lalanne, Gervasio et al. and Henry \& Buffat (see Roux 1990). Despite the limited resolution generally achieved, they all find that the three-dimensional flow is qualitatively different from the two-dimensional one, and that in addition, three-dimensional effects are significant in the entire cavity. In particular, Henry \& Buffat focus on the comparison between convective flows obtained for $A_{y}=1.0$ and $A_{y}=2.0$. They consider Prandtl numbers equal to 0 and $2.6 \times 10^{-2}$ and show that for $A_{y}=1.0$ the flow consists of a single circulation for all values of the Grashof number. On the other hand for $A_{y}=2.0$, a three-cell flow develops for increasing $G r$ and this is more in accord with the results of two-dimensional calculations. A more recent study by Henry \& Buffat (1998) concentrates on the $A_{y}=2.0$ geometry by drawing a detailed comparison with two-dimensional flows both for the steady and time-dependent regimes.

In contrast with the significant amount of analytical and numerical research discussed above, experimental work on convective flow in low Prandtl number fluids remains sparse. An early study of sidewall convection in molten gallium is reported by Hurle, Jakeman \& Johnson (1974). Their motivation is the control of striation growth in crystals and hence the investigation is primarily focused on the onset of time-dependent flow. However, they also find that in a cavity of aspect ratios $A_{x} \times A_{y}=2.7 \times 1.2$, the flow consists of a steady, single convective circulation for a relatively high value of the Grashof number of $1.0 \times 10^{5}$. This flow is clearly threedimensional in nature and in addition, it is left-right asymmetric. Hung \& Andereck (1988) discuss temperature measurements of the flow of mercury in a cavity of large aspect ratios equal to $A_{x} \times A_{y}=17.9 \times 17.8$. They find that the first transition is to stationary rolls in qualitative agreement with the weakly nonlinear stability analyses of Laure (1987) and Kuo \& Korpela (1988). A comparison is reported by Derebail \& Koster (1997) between x-ray visualizations of the temperature field in a cavity of aspect ratios $A_{x} \times A_{y}=1.4 \times 0.06$ and two- and three-dimensional numerical simulations which clearly shows that the convective flow is three-dimensional in this narrow geometry.

Braunsfurth et al. (1997) report a study of steady convective flow in cavities of 
aspect ratios $A_{x} \times A_{y}=3.0 \times 1.0$ and $4.0 \times 1.0$. Their results show that the flow appears to be primarily two-dimensional for small values of $G r$ by means of a direct comparison between experimental temperature measurements and one- and twodimensional models. In addition, they find that the variation of temperature across the width of the cavity is equal to approximately $3 \%$ of the applied temperature gradient which suggests that three-dimensional effects are small.

In comparative studies of a related problem undertaken by Schiroky \& Rosenberger (1984), Smutek et al. (1985) and Bontoux et al. (1986), the nature of free convective flow is investigated in a gas-filled, cylindrical cavity of aspect ratio 5.0, where the Prandtl number is $P r=7.3 \times 10^{-1}$. Using laser Doppler anemometry, Schiroky \& Rosenberger (1984) observe that part of the fluid diverges and drops along the sidewalls at a considerable distance from the cold endwall, thus generating a complex three-dimensional secondary flow. The three-dimensional numerical modelling of Smutek et al. (1985) confirms that complex three-dimensional structures are formed when the Rayleigh number exceeds $3.6 \times 10^{3}$. They do not, however, present a detailed description of the flow mechanisms involved. Finally, Bontoux et al. (1986) obtain excellent agreement between experimental measurements and three-dimensional numerical simulations over a wide range of Rayleigh numbers, whereas they find significant qualitative differences with two-dimensional analytical and numerical results both in the core-driven and the boundary-layer-driven regimes.

In this paper, we present a combined experimental and numerical study of the convective flow of liquid gallium in a differentially heated, rectangular enclosure of aspect ratios $A_{x} \times A_{y}=4.0 \times 1.0$. The model equations and mathematical techniques are briefly described in $\S 2$ and experimental details are presented in $\S 3$. In $\S 4.1$, a comparison between experimental measurements and two- and three-dimensional direct numerical simulations over an extended range of Grashof numbers clearly indicates the importance of three-dimensional flow mechanisms. Significant discrepancies between the spatial structure of the experimental flow and the three-dimensional simulations are also discussed. An interesting novel three-dimensional flow structure is described in $\S 4.2$, which is specifically observed for non-zero values of the Prandtl number and underpins the observed transition to time-dependence of the convective flow.

\section{Mathematical model and numerical techniques}

The mathematical model consists of a rectangular, insulating enclosure of square cross-section filled with gallium. It has aspect ratios $A_{x}=l / h=4.0$ and $A_{y}=w / h=$ 1.0, where $l$ is the length of the cavity, $h$ is its height and $w$ its width, as shown schematically in figure 1 . The endwalls are isothermal and held at $T_{\mathrm{c}}$ and $T_{\mathrm{h}}$, where the suffixes $\mathrm{c}$ and $\mathrm{h}$ correspond to cold and hot respectively. Thus, a horizontal temperature gradient is applied and this drives a convective circulation within the cavity.

We will refer to different cross-sections of the enclosure, in order to describe both the numerical and experimental flows. We denote by $X Y, Y Z$ and $X Z$ cross-sections the planes which are perpendicular to the $z-, x$ - and $y$-axes respectively. Examples of three cross-sections, centred on $x=A_{x} / 2, y=0$ and $z=0$ can be seen in figure 1 . These will commonly be referred to as the central cross-sections.

The free convection problem is modelled by the Navier-Stokes equations coupled to an energy equation and subject to the Boussinesq approximation. The characteristic scales chosen to non-dimensionalize the equations are $h, v / h, h^{2} / v, \rho v^{2} / h^{2}$ and 
$\gamma=\left(T_{\mathrm{h}}-T_{\mathrm{c}}\right) / A_{x}$, where $v$ is the kinematic viscosity and $\rho$ the density. They represent respectively length, velocity, time, pressure and temperature scales. Thus, in the usual notation, the equations take the following form:

$$
\begin{aligned}
\nabla \cdot \boldsymbol{u} & =0, \\
\frac{\partial \boldsymbol{u}}{\partial t}+(\boldsymbol{u} \cdot \nabla) \boldsymbol{u} & =-G r T \boldsymbol{g}-\nabla p+\nabla^{2} \boldsymbol{u}, \\
\frac{\partial T}{\partial t}+(\boldsymbol{u} \cdot \nabla) T & =P r^{-1} \nabla^{2} T,
\end{aligned}
$$

with boundary conditions

$$
\begin{gathered}
\partial T / \partial z=0 \quad \text { on } z= \pm 1 / 2, \quad \text { and } \quad \partial T / \partial y=0 \quad \text { on } y= \pm 1 / 2 \\
T=0 \quad \text { on } x=0, \quad \text { and } \quad T=A_{x} \quad \text { on } x=A_{x}
\end{gathered}
$$

and

$$
\boldsymbol{u}=\mathbf{0} \text { on all boundaries. }
$$

Under these conditions, the value of the pressure is not unique. Thus, its absolute value is set to zero at a convenient point within the cavity.

The scaling of the governing equations naturally leads to expressions for the Grashof number, $G r=\beta \gamma g h^{3} / v^{2}$ and the Prandtl number, $P r=v / \kappa$, where $\beta$ is the coefficient of thermal expansivity and $\kappa$ the thermal diffusivity.

The governing equations were computed on a three-dimensional domain using a spectral element method where the spatial discretization has been carried out using $31 \times 15 \times 15$ Gauss-Lobatto-Legendre collocation points. A detailed description of the methods and their implementation in MHD convection flows can be found in Ben Hadid \& Henry where the accuracy of the methods is also discussed. The twodimensional numerical simulations were run on a $32 \times 12$ mesh using the finite element method described by Braunsfurth et al. (1997).

\section{Experimental apparatus}

The experimental setup is similar to that described in Braunsfurth et al. (1997), and a schematic diagram of its central part is shown in figure 2. The sample of liquid gallium was held in an insulating rectangular channel which formed the bottom and lateral boundaries of the enclosure. It was machined in pyrophelite, a ceramic whose thermal conductivity is 29 times smaller than that of gallium. A non-conducting ceramic lid provided a rigid upper boundary. The channel was fitted between two $1 \mathrm{~mm}$ thick sheets of molybdenum, which is a good conductor and is also impervious to attack by gallium. Each endwall was the side of a copper box of capacity 0.71 , containing silicone oil, whose temperature was held constant to within $0.05^{\circ} \mathrm{C}$ by a commercial temperature controller. The convection cell was embedded within a layer of insulating material and the experiment was further enclosed in an air cabinet whose temperature was held constant at $32 \pm 0.5^{\circ} \mathrm{C}$. In particular, these precautions ensured that good uniformity and stability of the applied temperature at the two endwalls of the container were achieved.

An experimental procedure similar to that reported by Juel et al. (1999) was employed. The local temperature was measured with a type $\mathrm{K}$ insulated thermocouple of diameter $0.25 \mathrm{~mm}$ which was accurately positioned within the melt using a micromanipulator. The precision achieved in the measurement of relative temperatures was 


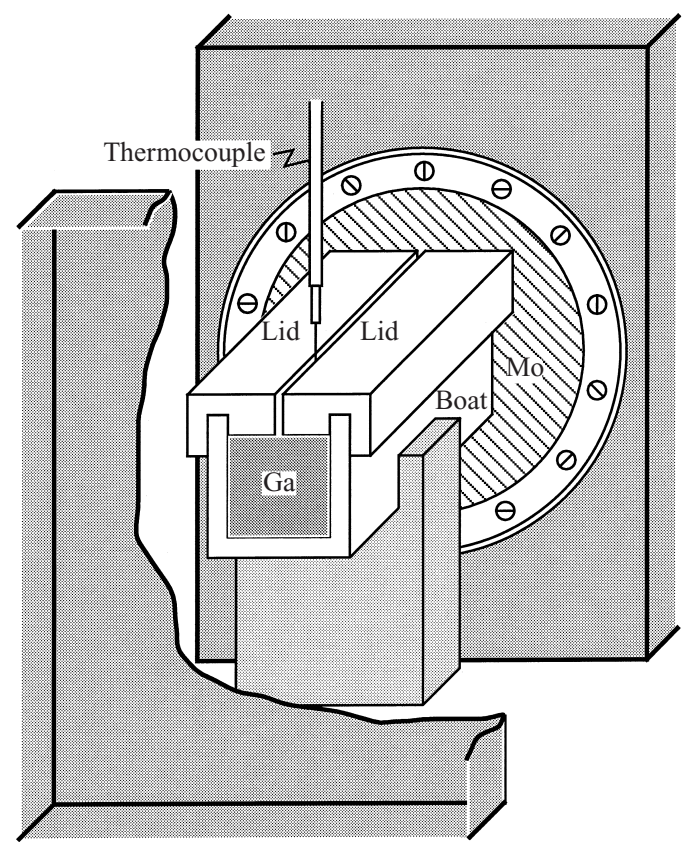

FIGURE 2. Schematic diagram of the central part of the experimental setup (from Braunsfurth et al. 1997). The inner dimensions of the convection cell are $50.8 \mathrm{~mm}$ in length, $12.7 \mathrm{~mm}$ in depth, and $12.8 \mathrm{~mm}$ in width.

better than $\pm 0.01^{\circ} \mathrm{C}$ and care was taken to ensure that the probe did not perturb the convective flow.

The vertical temperature difference $\Theta$, measured at a given location in the cavity, is of particular interest since it provides a measure of the amount of heat transferred by convection. Gallium is a metal and hence heat is mainly transported by conduction. The convective heat flow is relatively weak and is associated with motion of hot liquid in the top half of the container, progressing from the hot end to the cold, and vice versa in the bottom half. Convection is evident in the form of a moderate dependence of the temperature on the vertical coordinate $z$, which indicates thermal stratification. Estimates of $\Theta$ were obtained by measuring the amplitude of vertical temperature profiles which were sampled at selected locations within the cavity.

The steady flow investigation was carried out for mean temperatures of between $32.2^{\circ} \mathrm{C}$ and $40.6{ }^{\circ} \mathrm{C}$ and applied temperature differences of between $0.15^{\circ} \mathrm{C}$ and $10.6^{\circ} \mathrm{C}$, corresponding to Grashof numbers of between $9.0 \times 10^{3}$ and $6.9 \times 10^{4}$. The accurate determination of the absolute value of the experimental parameters relies on precise knowledge of the material properties in order to make quantitative comparisons with the numerical calculations. The data on the physical properties of molten gallium are sparse and their dependence on temperature is not well known. The available data were collected from a number of sources and discussed by Braunsfurth et al. (1997) as a function of temperature. At $37^{\circ} \mathrm{C}$, the thermal conductivity is $k=29.1 \pm 4 \mathrm{~W} \mathrm{~m}^{-1} \mathrm{~K}^{-1}$, the dynamic viscosity is $\eta=2.06 \times 10^{-3} \mathrm{~kg} \mathrm{~m}^{-1} \mathrm{~s}^{-1}$ and the density is $\rho=6.112 \times 10^{3} \mathrm{~kg} \mathrm{~m}^{-3}$. An average of the literature values for the specific heat of gallium yields $c_{p}=0.36 \pm 0.03 \mathrm{~kJ} \mathrm{~kg}^{-1} \mathrm{~K}^{-1}$ and the thermal expansion at $29.85^{\circ} \mathrm{C}$ is $\beta=1.3 \times 10^{-4} \mathrm{~K}^{-1}$. The Prandtl number is known to within a systematic uncertainty of $16 \%$ which is essentially due to lack of knowledge of the thermal 
conductivity. This systematic uncertainty is of little importance when comparing one set of experimental measurements with another, as the reproducibility of these results over a period of several months suggests that the sample of gallium retains its purity.

\section{Results}

\subsection{Comparison between the experimental flow and the numerical models}

The vertical temperature difference within the flowing layer of gallium was measured by recording vertical temperature profiles in the centre of the enclosure $(x=2.0$, $y=0$ ), for Grashof numbers up to $G r=6.9 \times 10^{4}$ and a Prandtl number of $\operatorname{Pr}=2.5 \times 10^{-2}$. This measurement is well suited for direct comparison with two- and three-dimensional numerical simulations of the idealized system.

The amplitude of the vertical temperature profiles is plotted as a function of the Grashof number in figure 3. The experimental data are shown as symbols, whereas the two- and three-dimensional numerical results are drawn with solid and dashed lines respectively. Most of the experimental data points for $\mathrm{Gr}$ up to $4.6 \times 10^{4}$ in figure 3 are taken from Braunsfurth et al. (1997) and all the higher values are new. The calibration of the experiment was subsequentially improved, and hence more accurate values of the Grashof number are presented in this paper, which are slightly different from those in figure 8 of Braunsfurth et al. (1997). The Prandtl number used in the calculations was set to $\operatorname{Pr}=2.5 \times 10^{-2}$. Calculations performed at $\operatorname{Pr}=3.0 \times 10^{-2}$ resulted in a similar Grashof number dependence, with the absolute values of the vertical temperature differences slightly higher in both the two- and three-dimensional cases. The three-dimensional result is included in figure 3 with a dotted line (the two-dimensional curve is omitted for clarity).

There is excellent qualitative agreement between the experimental data and those from the three-dimensional calculations for the dependence of convective heat transport on Grashof number. For values of the Grashof number smaller than approximately $G r \approx 3.0 \times 10^{4}$, the three-dimensional values of the vertical temperature difference are systematically below the two-dimensional ones. This is to be expected since the flow is bounded by the non-slip sidewalls, which damp the main convective circulation. All the numerical curves show an approximately linear dependence on the Grashof number, for $G r<1.0 \times 10^{4}$. However it should be noted that the two-dimensional slope is steeper due to higher velocities in the two-dimensional configuration. Hence, the importance of three-dimensional effects can be quantified even for very low values of the Grashof number.

The three-dimensional data intersect with the two-dimensional curve at approximately $G r \approx 3.0 \times 10^{4}$. Both sets of data then diverge when the Grashof number is further increased. This indicates that there are considerable qualitative differences between the flow fields in these two models. In the two-dimensional configuration, a transition from a parallel flow to one with co-rotating cells is found, whereas in the three-dimensional case, the flow consists of a large single convective circulation, for all the studied values of the Grashof numbers. Also, the strength of the convective flow saturates in the two-dimensional model whereas it continues to increase in both the experiment and three-dimensional numerical results. This increase for $G r>3.0 \times 10^{4}$ is interesting, since it clearly reflects the three-dimensional complexity of the steady flow. As will be discussed in $\S 4.2$, it results from the appearance of secondary cross-flows within the core of the cavity.

Although the experimental results shown in figure 3 qualitatively agree with the 




FiguRE 3. Vertical temperature difference in the centre of the enclosure $(x=2.0$ and $y=0)$ versus Grashof number. The experimental measurements are plotted with diamonds, the two- and three-dimensional numerical simulations for $\mathrm{Pr}=2.5 \times 10^{-2}$ are plotted with solid and dashed lines respectively. The dotted line corresponds to three-dimensional numerical data calculated for $\operatorname{Pr}=3.0 \times 10^{-2}$. The dots on each line are the points for which calculations were performed.

three-dimensional data, they mainly lie above the dashed line of numerical predictions. However, the three-dimensional calculation for $\mathrm{Pr}=3.0 \times 10^{-2}$ (dotted line) closely follows the experimental set of data and is therefore a good guide to the eye. The quantitative difference between experiment and three-dimensional calculations is larger than the uncertainties in the flow parameters such as the Prandtl number. Hence, its origin is to be sought in the difference between the ideal flow model computed and the real flow investigated in the laboratory.

The dependence of the vertical temperature difference on the lengthwise position within the cavity was next investigated. Whereas the steady model flows are axisymmetric (invariant by rotation of $\pi$ about the central $y$-axis), the experimental measurements exhibited an asymmetry such that the maximum vertical temperature difference was located at approximately $x=3.0$, as shown in figure 4 . Further measurements of time-averaged values of $\Theta$ for higher values of the Grashof number $\left(9.8 \times 10^{4}\right.$ and $1.3 \times 10^{5}$, where the flow was time-dependent and disordered) yielded the same dependence on $x$ as in figure 4 , hence confirming the robustness of the observed asymmetry.

Additional experiments showed that the location of the maximum vertical temperature difference, i.e. the centre of the convective circulation, was found to move when the ambient temperature of the surrounding cabinet was changed. Specifically, $\Theta_{\max }$ was shifted from $x=3.0$ to $x=1.0$ when the background temperature was 




FIGURE 4. Vertical temperature difference versus the lengthwise coordinate $x$ : comparison between experimental measurements for a background temperature of $32{ }^{\circ} \mathrm{C}$, equal to that of the cold endwall $\left(\mathrm{Gr}=4.2 \times 10^{4}\right.$ and $\left.\mathrm{Pr}=2.5 \times 10^{-2}\right)$ and two- and three-dimensional numerical simulations $\left(G r=4.0 \times 10^{4}\right.$ and $\left.P r=3.0 \times 10^{-2}\right)$.

raised by $\Delta T$ (on the order of $10^{\circ} \mathrm{C}$ ), from the temperature of the cold endwall to the temperature of the hot endwall. However, symmetry about $y=0$ was retained for all the values of the Grashof number shown in figure 3, for which the experimental flow was found to be steady.

These findings were all the more surprising as great care was taken in insulating the experimental convection cell from the ambient. Specifically, attempts were made to quantify heat losses on the outer walls of the enclosure with thermochromic liquid crystals and infrared measurements, but neither technique suggested a significant effect. Thus, the flow must be very sensitive to small imperfections in the thermal boundary conditions. On the other hand, the good qualitative agreement between numerical simulations and experiment for the dependence of convective heat transfer on Grashof number shows that the global features of the steady flow are robust. It is these aspects that are most likely to be of relevance in practical crystal growth facilities.

\subsection{Three-dimensional flow and transition to time-dependence}

The main convective circulation takes place in the plane of the two-dimensional geometry (central $X Z$-plane). In the three-dimensional simulations, the maximum transverse velocity $v$ is typically an order of magnitude smaller than the longitudinal component $u$. For $\mathrm{Pr}=2.5 \times 10^{-2}, v=6.69 \times 10^{-2}\left(3.07 \times 10^{-1}\right)$ for $\mathrm{Gr}=1.0 \times$ $10^{4}\left(7.0 \times 10^{4}\right)$ versus $u=7.03 \times 10^{-1}(1.22)$ and $w=3.59 \times 10^{-1}\left(6.48 \times 10^{-1}\right)$. Similarly, Braunsfurth et al. (1997) measured a transverse temperature variation 
(a)

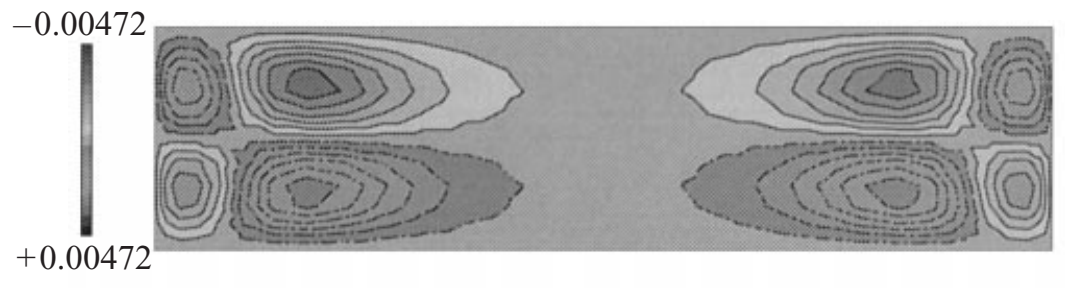

(b)

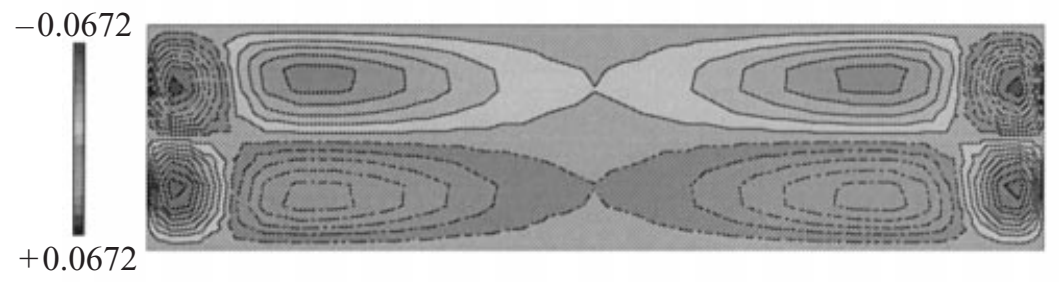

(c)

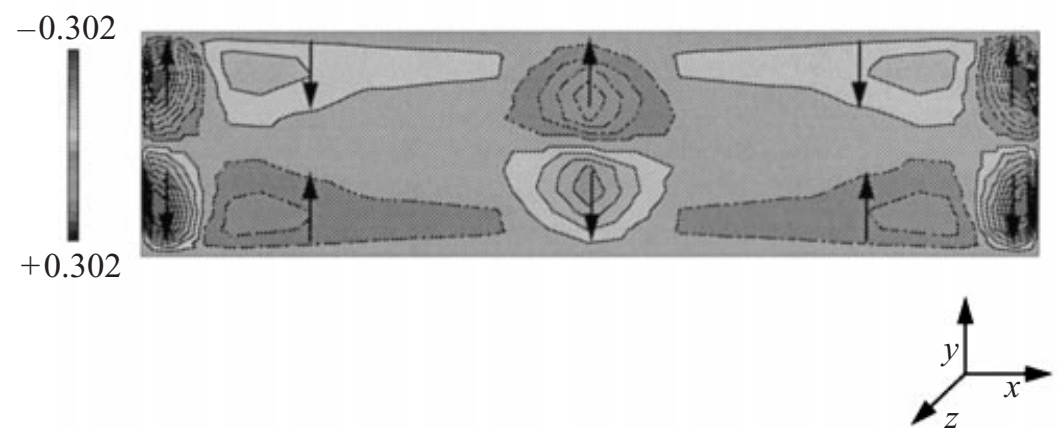

FIGURE 5. Plot of transverse velocity contours on the $X Y$-plane at $z=0$ : $(a) G r=1.0 \times 10^{3},(b)$ $G r=1.0 \times 10^{4}$ and $(c) G r=7.0 \times 10^{4}$. The Prandtl number is set to $\operatorname{Pr}=3.0 \times 10^{-2}$. In $(d)$ the arrows indicate the direction of the cross-flows.

of approximately only $3 \%$ of the applied temperature difference. Thus, one may be tempted to conclude that the flow in a confined enclosure is practically twodimensional in nature. However, the results of figure 3 show convincing evidence of the importance of three-dimensionality even for the smallest values of the Grashof number.

Evidence of three-dimensional cross-flows is primarily found close to each endwall, where the fluid particles in the upper layer, that are travelling from the hot to the cold end, progressively diverge towards the sidewalls, symmetrically on either side of the central $X Z$-plane as they approach the cold endwall (from approximately $x=0.1$ ). This results in a strong divergence of the flow in the $y$-direction in the upper two-thirds of the enclosure. Thus, the descending flow rate is larger on either side of the central $X Z$-plane than it is close to the centreline. This is because the velocity profile in the upper layer is close to parabolic so that the speed is larger in the central part of the cavity than close to the non-slip sidewalls. A stagnation point is formed halfway across the upper half of the cold endwall with a slight depression on either side of this point. Hence, a divergence of the fluid towards the sidewalls is favoured rather than a close approach of the endwall and a straight descent. The same mechanism is observed in the bottom layer at the cold endwall.

In figure 5, contours of transverse velocity $(v)$ are shown in the $X Y$-mid-plane, 
(a)



(c)

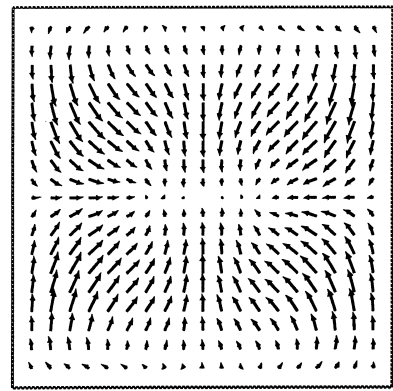

(e)

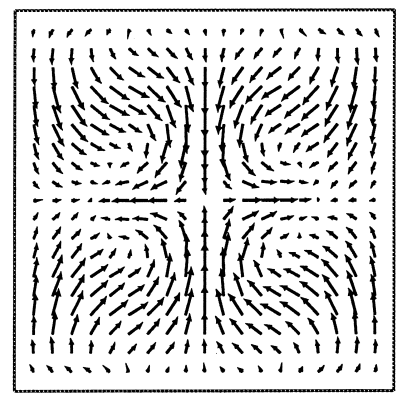

(b)

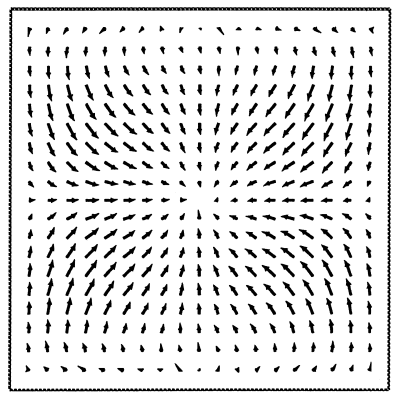

(d)

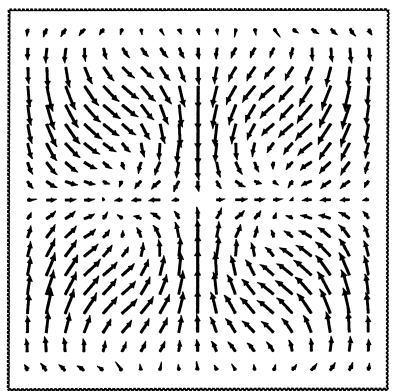

$(f)$

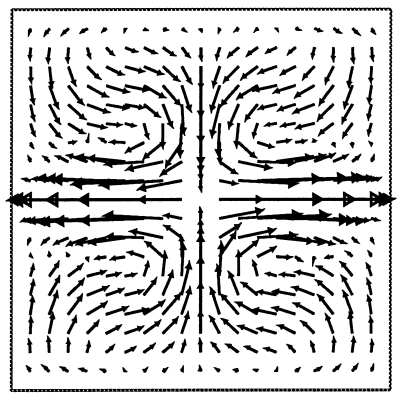

FIGURE 6. Plot of velocity vectors projected onto the $Y Z$-plane at $x=2.0:(a) G r=1.0 \times 10^{3},(b)$ $G r=1.0 \times 10^{4},(c) G r=2.0 \times 10^{4},(d) G r=3.0 \times 10^{4},(e) G r=5.0 \times 10^{4}$ and $(f) G r=7.0 \times 10^{4}$. The Prandtl number is set to $\mathrm{Pr}=3.0 \times 10^{-2}$.

viewed from above, for three values of the Grashof number, $G r=1.0 \times 10^{3}, 1.0 \times 10^{4}$ and $7.0 \times 10^{4}$. The dashes (solid) contours correspond to negative (positive) velocities respectively. The regions of divergent flow in the vicinity of the endwalls are clearly observed in all three cases, including for the very small value of $G r=1.0 \times 10^{3}$. However, cross-flows are not restricted to the close vicinity of the endwalls and evolve significantly as the Grashof number is raised. Between $\mathrm{Gr}=1.0 \times 10^{3}$ and $G r=1.0 \times 10^{4}$, regions of convergent flow, initially adjacent to the divergent flows at the endwalls, extend into the centre of the cavity. In fact these convergent flows which underpin global spiralling motions from the sidewalls towards the central $X Z$-plane were previously reported by Henry \& Buffat (1998). For $G r=1.0 \times 10^{4}$, the cross-flows from the cold and hot halves of the enclosure have spread to the centre of the $X Y$-mid-plane and the strengthening of the interaction between these convergent flows leads to the appearance of a central region of divergent flow for 

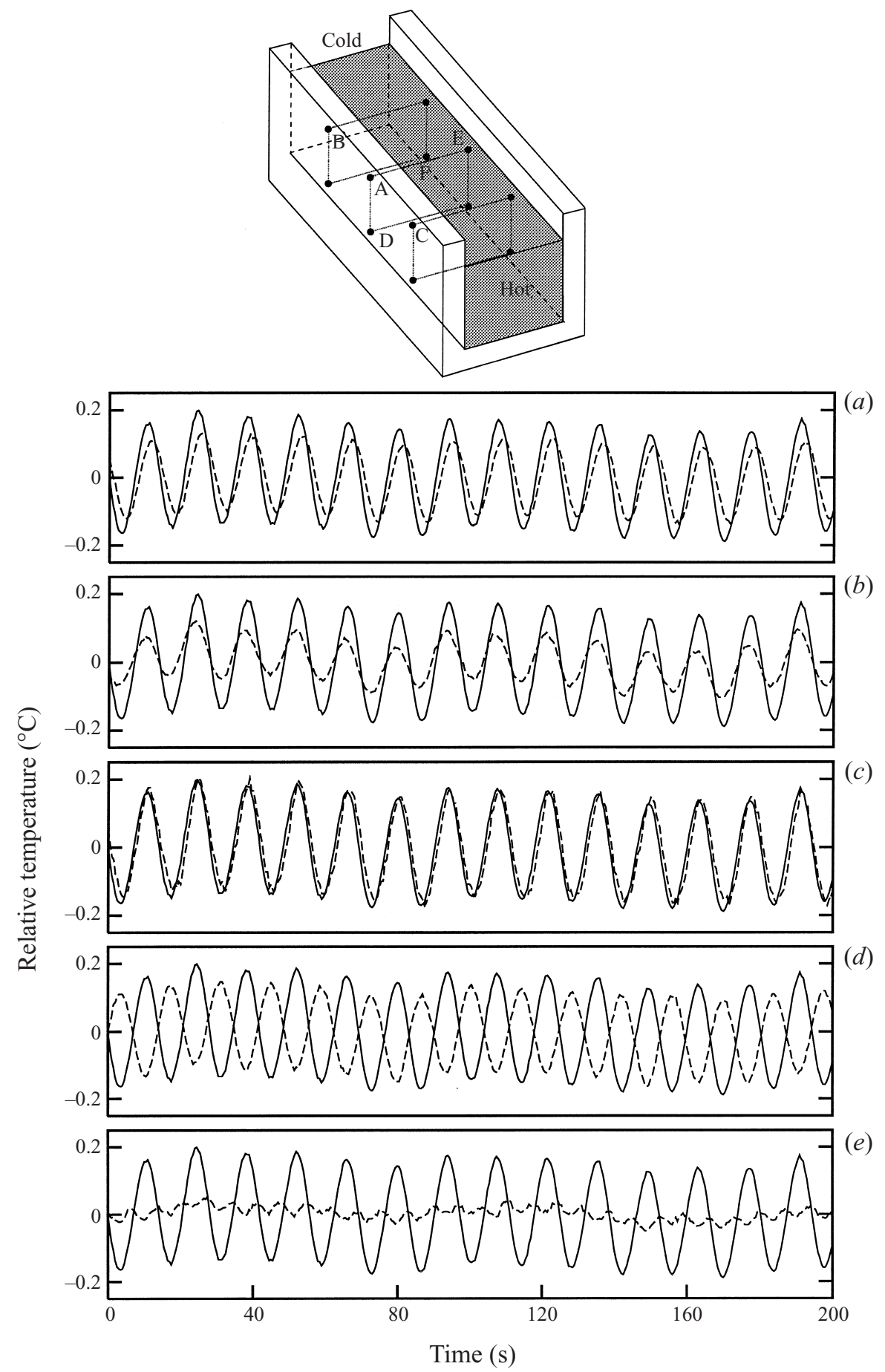

FIGURE 7. For caption see facing page.

$G r=7.0 \times 10^{4}$. Although the flow retains its large, single circulation in the plane of the two-dimensional geometry (central $X Z$-plane), the complex cross-flow dynamics mentioned above are indicated by a localized, central region of shear.

A detailed sequence of the above transition is shown in figure 6 with a set of 
velocity fields calculated for Grashof numbers between $G r=1.0 \times 10^{4}$ and $7.0 \times 10^{4}$, that are projected onto the central $Y Z$-plane. The cross-flows can then be clearly observed since the main circulation is in the $x$-direction. The same scale is used in each plot. The second-order transverse flow predicted by Bejan \& Tien (1978b) is only observed for very small values of the Grashof number on the order of $G r=1.0 \times 10^{3}$. For $G r=1.0 \times 10^{4}$, the convergent flows described above have spread into the centre, so that the vectors are all pointing towards the centre point of the $Y Z$-mid-plane. Of course, it should be remembered that the plot is a projection, which actually depicts a three-dimensional flow process with a strong velocity component in the $x$-direction. Also, the projected velocity field can be separated into four symmetric quadrants, separated by horizontal and vertical velocity vectors which are oriented towards the centre. When the Grashof number is raised to $G r=2.0 \times 10^{4}$, the form of the velocity distribution does not change although the mean velocity of the cross-flow increases. For $\mathrm{Gr}=3.0 \times 10^{4}$, the flow along the vertical boundary line remains oriented towards the centre. On the horizontal line however, the velocity vectors are now mainly directed towards the centre, although close to the mid-point a weak divergent flow has appeared. Thus a qualitative change in the velocity distribution on the central $Y Z$-plane has taken place between $G r=2.0 \times 10^{4}$ and $G r=3.0 \times 10^{4}$. This can be observed in figures $6(d)$ and $6(e)$, where the divergent flow is found to strengthen and also develops across the width of the cavity. This results in the creation of a three-dimensional hyperbolic point in the centre of the $Y Z$-plane. Finally, in figure $6(f)$ for $G r=7.0 \times 10^{4}$, the divergent horizontal flow occupies the entire width of the cavity and it has also spread vertically. Furthermore, it clearly dominates the three-dimensional recirculation structure at this location.

The fundamental role of the cross-flows in the structure of the convective flow is further emphasized in the transition to time-dependence. Oscillatory convection is observed for $\mathrm{Gr}=8.0 \times 10^{4}$ and $\mathrm{Pr}=2.5 \times 10^{-2}$, where the central divergent cross-flow structure depicted in figures $6(c)$ and $6(f)$ is destabilized to an oscillatory motion along the length of the cavity.

Although the coupling between flow field and temperature field is weak, it still plays an essential role in determining the nature of the flow, since the convective dynamics computed for $\mathrm{Pr}=2.5 \times 10^{-2}$ and $\mathrm{Pr}=0$ are fundamentally different. For $P r=0$, a complicated cross-flow structure is also formed but it remains for the most part localized in the endwall regions, so that interactions between crossflows halfway along the length of the enclosure are negligible. Thus, the divergent cross-flow structure seen in figures $6(c)$ and $6(f)$ is not formed, and a time-dependent calculation for $G r=8.5 \times 10^{4}$ indicates that oscillations of the three-dimensional flow are primarily in the transverse $(y)$ direction (see Henry \& Buffat 1998 for related results on the $4 \times 2 \times 1$ enclosure). Hence, it is essential to simulate the convective

FIGURE 7. Top: Schematic of the sampling locations: A, B, C, D, E and F are located at $(x, y, z)=(2.0,0.5,0.4),(1.0,0.5,0.4),(3.0,0.5,0.4),(2.0,0.5,-0.3),(2.0,-0.5,0.4)$ and $(2.0,0,0.4)$ respectively. Below: Comparison between extracts of time series recorded at A (solid line) and other locations (dashed line): (a) B, (b) $\mathrm{C},(c) \mathrm{D},(d) \mathrm{E}$ and $(e) \mathrm{F}$. The flow parameters are $\mathrm{Gr}=5.44 \times 10^{4}$ and $\operatorname{Pr}=1.97 \times 10^{-2}$. Note that the critical Grashof number in this experiment $\left(G r_{\mathrm{c}}=5.37 \times 10^{4}\right)$ is small compared with that estimated from the three-dimensional numerical simulations (between $G r_{\mathrm{c}}=7.0 \times 10^{4}$ and $8 \times 10^{4}$ ) for $\mathrm{Pr}=2.5 \times 10^{4}$ and $A_{y}=1.0$. This is to be expected since the value of $P r$ is reduced in this experiment and $A_{y}$ is increased to 1.3. The phase relationships exhibited in these plots suggest that the experimental oscillations at onset correspond to a standing wave across the width of the enclosure. 
flow at finite values of the Prandtl number if comparison with experiment is to be made.

Interestingly, in the experiment a robust onset of singly periodic oscillations could not be achieved in the $4 \times 1 \times 1$ enclosure, and a direct transition from steady flow to intricate and noisy dynamics was observed. However, when the width to depth ratio, $A_{y}$, was raised to 1.3 by increasing the thickness of the lid (which also raised $A_{x}$ to 5.0), the transition to time-dependence took place through a simple Hopf bifurcation. Extracts of time series are shown in figure 7, that were sampled close to onset in several locations along the walls of the enclosure such as to minimize disturbances on the flow. The location of all the thermocouple probes are shown schematically in the top part of figure 7 . The signal sampled at location A, halfway along the length of the cavity consistently had the largest amplitude for increasing values of the Grashof number. All the temperature traces sampled on the same sidewall (at locations B, C and $\mathrm{D}$ ) are in phase with $\mathrm{A}$, whereas the signal sampled on the opposite sidewall, at location $\mathrm{E}$, is $\pi$ out of phase with $\mathrm{A}$. In addition, the signal at location $\mathrm{F}$, halfway across the width of the enclosure, is an order of magnitude smaller than for the sidewall locations, and approximately $\pi / 2$ out of phase with A. Hence, the observed oscillations correspond to a transverse, standing wave, with at least one central node.

The evidence presented in this section points to the importance of three-dimensional effects in the transition to oscillatory flow in both the experiment and the numerical simulations, although the nature of the oscillations is different in each case. However, such a discrepancy is to be expected, since the transition to oscillatory flow takes place for relatively high Grashof numbers, in a regime where the bulk flow is fully established. Thus, the flow mechanisms are strongly nonlinear, so that small differences between the experiment and the model can be substantially amplified.

\section{Conclusion}

We have provided convincing numerical and experimental evidence for the importance of three-dimensional effects in free convective flow of liquid gallium in a rectangular enclosure of moderate aspect ratios. Agreement has been demonstrated between experimental measurements and three-dimensional simulations of the convective heat transfer over an extended range of Grashof numbers, for which the convective flow is steady. Complex three-dimensional flow processes were shown to be essential in establishing the steady flow for all values of the applied temperature gradient, although the intensity of the cross-flows was typically of an order of magnitude smaller than the main two-dimensional circulation. Additional numerical calculations suggested the complex flow structure uncovered in the centre of the cavity to be of great importance in the transition to time-dependent flow, and the onset of oscillations in the experiment took the form of a standing wave across the width of the enclosure.

The work of A. J. was supported by a studentship funded by DERA Malvern and the collaborative research was supported by the British Council under the Alliance Program.

\section{REFERENCES}

Bejan, A. \& Tien, C. L. 1978 a Laminar natural convection heat transfer in a horizontal cavity with different end temperatures. Trans. ASME: J. Heat Transfer 100, 641-647.

Bejan, A. \& Tien, C. L. $1978 b$ Fully developed natural counterflow in a long horizontal pipe with different end temperatures. Intl J. Heat Mass Transfer 21, 701-708. 
Ben Hadid, H. \& Henry, D. 1997 Numerical study of convection in the horizontal Bridgman configuration under the action of a constant magnetic field. Part 2. Three-dimensional flow. J. Fluid Mech. 333, 57-83.

Bontoux, P., Roux, B., Schiroky, G. H., Markham, B. L. \& Rosenberger, F. 1986 Convection in the vertical midplane of a horizontal cylinder. Comparison of two-dimensional approximations with three-dimensional results. Intl J. Heat Mass Transfer 29, 227-240.

Braunsfurth, M. G., Skeldon, A. C., Juel, A., Mullin, T. \& Riley, D. S. 1997 Free convection in liquid gallium. J. Fluid Mech. 342, 295-314.

Cormack, D. E., Leal, L. G. \& Imberger, J. 1974 Natural convection in a shallow cavity with differentially heated endwalls. Part 1. Asymptotic theory. J. Fluid Mech. 65, 209-229.

Daniels, P. G., Blythe, P. A. \& Simpkins, P. G. 1987 Onset of multicellular convection in a shallow laterally heated cavity. Proc. R. Soc. Lond. A 114, 327-350.

Derebail, R. \& Koster, J. N. 1997 Numerical simulation of natural convection of gallium in a narrow gap. Intl J. Heat Mass Transfer 40, 1169-1180.

GiLl, A. E. 1974 A theory of thermal oscillations in liquid metals. J. Fluid Mech. 64, 577-588.

HarT, J. 1972 Stability of thin non-rotating Hadley circulations. J. Atmos. Sc. 29, 687-697.

HART, J. 1983a Low Prandtl number convection between differentially heated endwalls. Intl J. Heat Mass Transfer 26, 1069-1074.

HarT, J. 1983b A note on the stability of low-Prandtl-number Hadley circulations. J. Fluid Mech. 132, 271-281.

Henry, D. \& Buffat, M. 1998 Two- and three-dimensional numerical simulations of the transition to oscillatory convection in low-Prandtl-number fluids. J. Fluid Mech. 374, 145-171.

Hill, P. 1998 Crystal growth meets demands of opto devices. Opto \& Laser Europe 56, 33-35.

Hung, M. C. \& ANDERECK, C. D. 1988 Transitions in convection driven by a horizontal temperature gradient. Phys. Lett. A 132, 253-258.

Hurle, D. T. J., Jakeman, E. \& Johnson, C. P. 1974 Convective temperature oscillations in molten gallium. J. Fluid Mech. 64, 565-576.

Juel, A., Mullin, T., Ben Hadid, H. \& Henry, D. 1999 Magnetohydrodynamic convection in molten gallium. J. Fluid Mech. 378, 97-118.

Kuo, H. P. \& Korpela, S. A. 1988 Stability and finite amplitude natural convection in a shallow cavity with insulated top and bottom and heated from a side. Phys. Fluids 31, 33-42.

LAURE, P. 1987 Study of convective motions in a rectangular cavity with horizontal gradient of temperature. J. Méc. Théor. Appl. 6, 351-382.

Müller, G. \& Ostrogorsky, A. 1993 Convection in melt growth. Handbook of Crystal Growth, vol. $2 b$ (ed.) D. T. J. Hurle. North-Holland.

Roux, B. 1990 GAMM workshop 1990: Numerical simulation of oscillatory convection in low $\operatorname{Pr}$ fluids. Notes on Numerical Fluid Mechanics, vol. 27. Vieweg.

Schiroky, G. H. \& Rosenberger, F. 1984 Free convection of gases in a horizontal cylinder with differentially heated endwalls. Intl J. Heat Mass Transfer 27, 587-598.

Smutek, C., Bontoux, P., Roux, B., Schiroky, G. H., Hurford, A. C., Rosenberger, F. \& Vahl DAVIS, G. DE 1985 Three-dimensional convection in horizontal cylinders: numerical solutions and comparison with experimental and analytical results. Num. Heat Transfer 8, 613-631. 